\title{
Channel Adaptation for Time-varying Powerline Channel and Noise Synchronized with AC Cycle
}

\author{
Kyong-Hoe Kim ${ }^{1}$, Han-Byul Lee ${ }^{1}$, Yong-Hwa Kim ${ }^{2}$, and Seong-Cheol Kim ${ }^{1}$ \\ ${ }^{1}$ Institute of New Media and Communications, \\ School of Electrical Engineering and Computer Science \\ Seoul National University, Seoul, Korea \\ \{khkim, hblee, sckim\}@maxwell.snu.ac.kr \\ ${ }^{2}$ Korea Electrotechnology Research Institute (KERI), Ansan-city, Gyeonggi-do, Korea \\ yongkim@keri.re.kr
}

\begin{abstract}
Broadband Power Line Communications (PLC) technology allows the usage of electrical power supply networks for communications purposes, such as audio/video streaming and broadband internet access. The powerline has an inherent characteristic that is the cyclic variation of channel and noise with the phase of the AC line cycle. To achieve either high throughput or high robustness, we apply dynamic channel adaptation scheme that exploits the cyclic variation of powerline characteristics. In this paper, we introduce an expedient, dynamic channel adaptation which is robust against the short-time variation of the AC synchronized powerline channel and noise. We analyze the performance enhancement for the measured powerline channel and noise when the proposed adaptation scheme is applied to the Korean standard on high speed powerline communications.
\end{abstract}

Keywords-channel adaptation, channel measurement, LPTV, powerline communication.

\section{INTRODUCTION}

$I^{\top}$ recent years, broadband Power Line Communication (PLC) systems have drawn a huge amount of research interest to develop high performance, yet cost efficient, digital data transmission systems. Nowadays, broadband PLC systems are considered as an inexpensive and effective alternative to Digital Subscriber Line (DSL), cable and wireless technologies. However, powerline conductors were primarily designed for transmission of power at 50 or $60 \mathrm{~Hz}$, not for communication purposes. Moreover, characteristic impedances of the powerline vary based on the conductor type as well as a function of frequency. One of the most inevitable phenomenons in power line networks is impedance mismatch, which result in a multipath effect that often cause deep notches in certain frequency bands. Electrical appliances connected to the power line also significantly impact the PLC channel characteristics.

Unlike the other telecommunications channels, the powerline channel does not represent an Additive White Gaussian Noise (AWGN). Noises in the powerline can be classified mainly into two categories, namely the background noise and the impulsive noise. The background noise has a power spectral density which decreases with frequency, and is usually stationary over relatively longer periods, of seconds,

This work is supported by Brain Korea 21 project. minutes and sometimes even of some hours. Because of its higher amplitudes, impulsive noise is considered the main cause of burst error occurrence in data transmission over the high frequencies of the powerline medium. One of interesting characteristics of the impulsive noise is its cyclic dependency on the phase of the underlying AC line cycle. This type of noise is often referred to periodic impulsive noise, synchronous to the main frequency [1].

The cyclic nature of noise in power line networks has been recently known by the scientific community. Authors in $[2,3]$ were among the first to characterize the noise in $(0-500 \mathrm{kHz})$ frequency band as 'cyclo-stationary'. The cyclo-stationary noise characteristics in the band from $\mathrm{DC}$ to $25 \mathrm{MHz}$ were investigated in [4]. On the other hand, the time-varying channel characteristics are also synchronized with the AC line cycle. Authors in [5] proposed a Linear Periodically Time Varying (LPTV) model as the PLC channel. Extensive channel characterization measurements presented in [6] show time-varying channel response synchronized with AC powerline. The frequency response of powerline channel alternates two different frequency responses according to the phase of the underlying AC line cycle.

Through the measurement results presented in the above literature, we can easily derive that the performance enhancements can be achieved by using a channel adaptation mechanism that exploits the cyclic variation in powerline channel and noise.

In this paper, we study the performance enhancement by using dynamic channel adaptation where the effect of the cyclic variation exists in both the channel characteristic and the noise figure. The Korean standard on high speed powerline communications published in 2006 [7] has no consideration about AC synchronized variation. We introduce an expedient, dynamic channel adaptation which is robust against the short-time variation of the $\mathrm{AC}$ synchronized powerline channel and noise. Eventually, we analyze the performance enhancement when the proposed adaptation scheme is applied to the Korean standard on high speed powerline communications.

The rest of the paper is structured as follows. Section II describes the measurement results which are used in the simulation. Dynamic channel adaptation scheme over cyclic 
channels is presented in Section III. The system model used for the simulation is described in Section IV. In Section V, the performance evaluation based on simulations for various scenarios is presented. Finally, the paper will be summarized in Section VI.

\section{CHANNEL AND NOISE MEASUREMENTS}

For this study, channel transfer functions and time domain noise captures spanning couples of $60 \mathrm{~Hz}$ AC line cycle were measured at a typical in-home powerline. To measure very short time variation of the channel transfer function and the noise, which is only subject to the amplitude of mains voltage, measurements must be conducted in a static environment. In other words, to capture the sole effect of LPTV channel, changes in the powerline network must be from mains voltage variation (60 Hz in Korea), not from switching of appliances and/or any other disturbances in the powerline. To meet this condition, we measured the channel and the noise at the powerline experimental station in Daejeon, Korea. The powerline experimental station was built by Korea Electric Power Research Institute (KEPRI) in 2007. In the powerline experimental station, there is a PLC home network test house which reproduces a typical residential house. So, there are many kinds of home appliances, cables, lamps which are typically used and installed in the real home. All these electrical devices can be totally controlled by the software. Moreover, this test house is electrically isolated from adjacent houses. These limited and controllable features of the test house make the measurement for LPTV channel and short time varying noises ideal. Measurements were fulfilled only for the purpose of the simulation described in Section V, and the modeling of the cyclo-stationary nature of the powerline channel and noise is beyond the scope of this paper.

Channel transfer functions up to $50 \mathrm{MHz}$, that includes a typical frequency band for broadband PLC, are measured by using a measurement system described in Fig. 1. The measurement system consists of Arbitrary Waveform Generator (AWG) as a transmitter and Digital Storage Oscillator (DSO) as a receiver. The AWG, Tektronix AFG3101 was loaded with a sounding waveform sampled at 100 MSample/sec by using Tektronix software Arbexpress. The sounding waveform was designed for the channel transfer function estimation as a repeating OFDM symbol which has flat frequency spectrum and low Peak-to-Average Power Ratio (PAPR). The number of subcarriers is 2048. The signal duration of the repeating OFDM symbol is $20.48 \mu \mathrm{s}$. So, there are about 813 OFDM symbols in an AC cycle. The transmitting signal passed through TX coupler, powerline channel, and RX coupler in sequence. At the receiver side, the decoupled signal was digitalized and recorded by the DSO. Received data is transferred from the DSO to the PC, where the signal processing calculation is done for the channel estimation. The algorithm for channel estimation is widely known $[5,6]$ and omitted here.

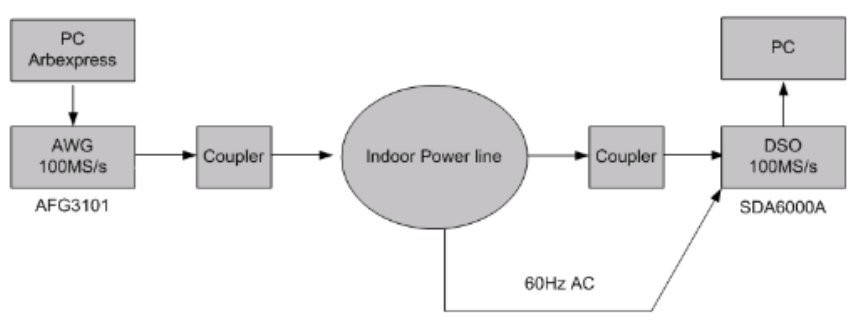

Fig. 1. Block diagram of channel transfer function measurement system

The DSO, LeCroy SDA6000A can record the received signal up to 16 Msample points at the sampling rate 100 MSample/sec. Therefore, with a single measurement sweep, we can record the received signal for $160 \mathrm{~ms}$, about 9 cycles of 60 $\mathrm{Hz} \mathrm{AC}$ mains. With the assumption that the circumstance of the test house is electronically static, $9 \mathrm{AC}$ line cycles are enough to measure the LPTV channel property. From comprehensive measurement results, we confirmed that there were negligible changes between 9 channel frequency responses of each $\mathrm{AC}$ line cycle.

There are another connection between the AC powerline and the DSO. The AC powerline is connected to the DSO via a 200:1 differential probe. This probe attenuates input signal to $1 / 200$ ratio. Therefore, $220 \mathrm{~V} A C$ is attenuated to $1.1 \mathrm{~V}$ sinusoidal waves which are acceptable to the DSO. This signal is used for the channel and noise measurement as a timing reference. So, with the help of the reference AC signal, we can observe how the shapes of the channel and noise are changed at a specific phase of an AC cycle.

Fig. 2 shows the measured AC mains signal as a timing reference and Fig. 3 illustrates the amplitude spectrum of channel frequency responses with respect to AC cycle. Fig. 4 shows the measured powerline noise. The horizontal axes of Fig. 2, 3, and 4 are synchronized each other. A 3-dimensional plot of Fig. 3 is described in Fig. 5.

It is easily observed that the channel frequency responses from the measurement alternate between two kinds of frequency response according to the AC mains voltage and abrupt transition between them. This LPTV phenomenon is very similar to the measurement result of preceding research in [6]. The channel transfer function $H(t, f)$ can be represent by

$$
H(t, f)=\left\{\begin{array}{ll}
H_{\text {narrow region }}(f), & \text { if }|v(t)| \leq V_{\text {thr }} \\
H_{\text {wide region }}(f), & \text { if }|v(t)|>V_{\text {thr }}
\end{array},\right.
$$

for AC mains voltage $v(t)$, where $V_{\text {thr }}$ represents some threshold and the subscript 'narrow region' and 'wide region' is named from the duration of channel transfer function.

Actually, it is not common for the in-home powerline. But, if some kind of appliances (loads) is connected to the outlet nearly located in the receiver, the LPTV situation appears dominantly as shown in Fig. 3. It is known that those loads have two states of impedance value are probably caused by silicon control rectifiers and the impedance seems to depend on the absolute level of the mains signal, resulting in a periodicity of the channel transfer function [8]. 




Fig. 2. Measured AC mains signal

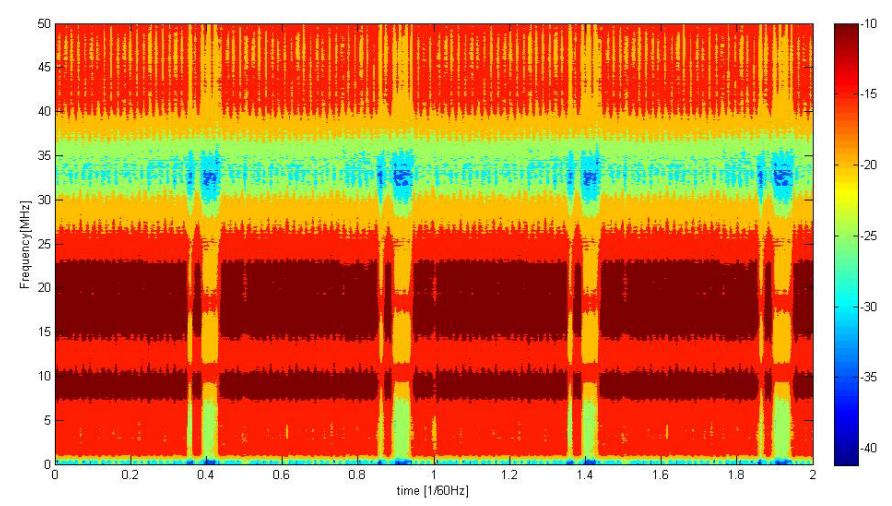

Fig. 3. Amplitude spectrum of channel frequency responses (in dB scale)

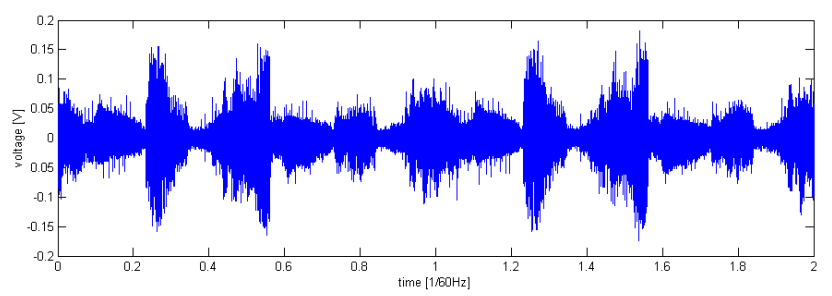

Fig. 4. Measured powerline noise

We decided to use snapshots of alternating channel transfer functions for the simulation. The powerline noise synchronized with AC mains in Fig 4 is also used for the simulation. Two snapshots of alternating channel transfer functions referred to 'narrow region' and 'wide region' are illustrated in Fig. 6.

Time domain noise was measured by using the DSO. Time domain noise was sampled at $100 \mathrm{M}$ samples/sec spanning couples of $60 \mathrm{~Hz}$ AC line cycle. The measurement result illustrated in Fig. 4 shows that the powerline noise characteristics vary as a function of one or a half $\mathrm{AC}$ line cycle $(60 / 120 \mathrm{~Hz})$. As the noise power varies with the phase of the underlying AC line cycle, a SNR at the receiver also varies with the AC line cycle.

From these results, one can deduce that the channel adaptation for periodically time varying PLC channels should optimize the system performance. The proposed dynamic channel adaptation scheme over cyclic channels is presented in the following section.

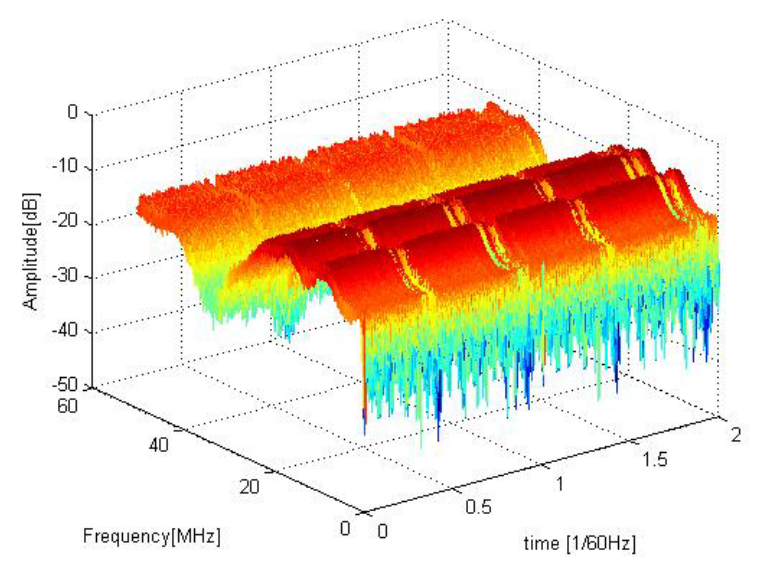

Fig. 5. 3-dimensional plot of amplitude spectrum

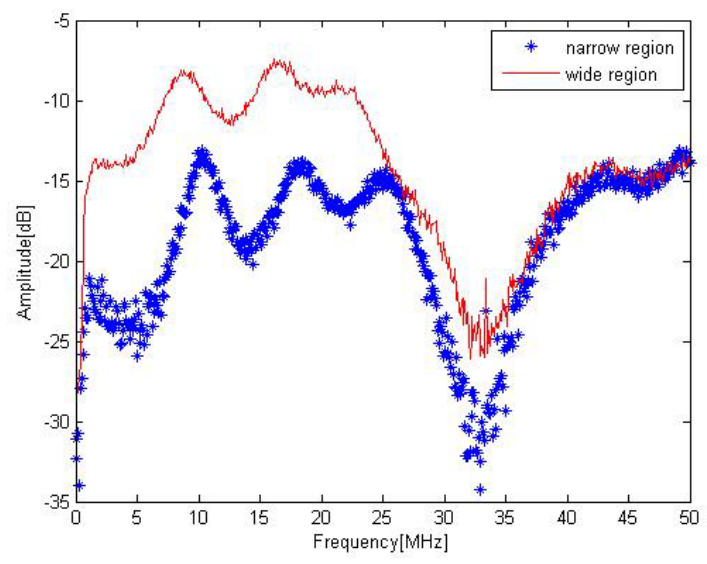

Fig. 6. Snapshots of the measured channel transfer function

\section{CHANNEL ADAPTATION}

To overcome unfavorable channel properties of powerline transmission medium, most of broadband PLC systems in these days have to adopt efficient multi-carrier modulation techniques such as Orthogonal Frequency Division Multiplexing (OFDM). Channel adaptation in these PLC systems enables fine tuning of the PHY parameters to optimize the system performance. Channel adaptation is mainly accomplished by Adaptive Modulation Coding (AMC) using bit-loading algorithms. Therefore, the PHY parameters mentioned above include modulation scheme, transmitting power distribution, and coding rate, etc.

For periodically time-varying PLC channels, channel adaptation should also determine the dependency of the PHY parameters on the phase of the AC line cycle. One simple method to fulfill the channel adaptation is by dividing the $\mathrm{AC}$ line cycle into multiple temporal adaptation windows and determining the PHY parameters independently for each window.

Note that there is an important trade-off between the number of windows and the system performance. As the number of windows increases, a finer adaptation to temporal periodic channel characteristics can be obtained. So, this enhances the system performance. However, this also increases the complexity of the system. A larger number of windows require the transmitter and receiver to determine and maintain a 
larger set of PHY parameters and to apply them with higher accuracy within the AC line cycle. The bandwidth and memory requirements for generation, storing and exchanging the PHY parameters can be massively expensive. So, it is important to determine the appropriate number of windows according to the system capacity.

To learn and adapt the cyclic variation of the powerline channel and noise, we propose the channel adaptation process described in the following steps.

1. For the whole AC line cycle (16.66 ms) or more, the receiver scans the powerline channel by using sounding preamble sent by the transmitter.

2. The receiver writes Tone Maps which is calculated according to the bit-loading algorithm and used for each pre-defined temporal window. A Tone Map includes the PHY parameters of each subcarrier. Tone Maps are sent to the transmitter via a feedback channel.

3. The transmitter assigns the PHY parameter such as modulation scheme, transmitting power, and coding rate to each subcarrier for pre-defined temporal window. Then, the transmitter generates and sends AC line cycle adapted signals which suit assigned Tone Maps for each pre-defined temporal window.

4. If the Tone Map is deemed to be invalid (for example, due to high frame error rates), the receiver may provide a new Tone Map to replace the existing Tone Map or request to process the channel sounding (that is, back to the step 1) again.

\section{System Model}

To investigate the channel adaptation scheme proposed in Section 3, we introduce broadband hi speed PLC system based on the Korean standard [7]. The PHY specification of the standard defines Discrete Multi-Tone (DMT) as a modulation scheme. DMT is an application of OFDM for a wired channel with efficient bit-loading techniques. The basic idea of DMT is to divide the available bandwidth into a fixed number of $N$ parallel, independent, and equally spaced subchannels. A detailed description of the PHY specification is shown in Table I.

First we divide the $60 \mathrm{~Hz}$ AC line cycle into multiple equally separated windows. Therefore, there are multiple numbers of DMT symbols in each window. In each window, the receiver calculates a Tone Map according to the bit-loading algorithm. The Tone Map can be derived from the averaged value of the channel characteristics within the window. The bit-loading algorithm and LDPC codes used in this simulation is described well in our previous work [9]. The rest of the process is followed as the proposed channel adaptation scheme described in Section III.

We deal with another scenario for the performance comparison. The system in this scenario is the same as the proposed system except that there is only a single Tone Map for the transmitter and the receiver. For the calculation of the Tone TABLE I

PHY SPECIFICATION OF THE KOREAN PLC STANDARD

\begin{tabular}{|l|l|}
\hline Bandwidth & $2 \sim 28 \mathrm{MHz}$ \\
\hline Tone Separation & $24.4141 \mathrm{kHz}$ \\
\hline Sampling Frequency & $62.5 \mathrm{MHz}$ \\
\hline IFFT Interval & 2560 samples, 40.96 $\mu \mathrm{sec}$ \\
\hline Cyclic Prefix Interval & 892 samples, 14.272 $\mu \mathrm{sec}$ \\
\hline Tone Modulation & BPSK, 4-QAM, 16-QAM, \\
& 64-QAM, 256-QAM, 1024-QAM \\
\hline
\end{tabular}

Map, the receiver randomly captures any phase of $\mathrm{AC}$ line cycle at each a simulation run. That means there is no consideration about the channel adaptation for periodically time-varying PLC system within $\mathrm{AC}$ line cycle (i.e., no $\mathrm{AC}$ line cycle based adaptation).

\section{Simulation Results}

In this section, the performance of the proposed system is compared to the system which don't use AC line cycle based adaptation. It is expressed both in terms of throughput (bit rate) and BER derived by simulations under the assumptions of AC synchronized powerline channel and noise.

For the simulation comparison, we introduce different scenarios according to the time duration of adaptation window. If the time duration of window is equal to a DMT symbol duration, we call this scenario to a full adaptation. As described in Section III, in the full adaptation scenario, we can adapt the LPTV powerline channel with the finest granularity at the expense of the system complexity. If the time duration of window is equal to multiple DMT symbol duration, we call this scenario to a partial adaptation. In the partial adaptation, the number of windows in an AC line cycle is key parameter which determines the trade-off between the performance and the system complexity. The last is no adaptation scenario which don't use AC line cycle based adaptation.

Measured channel transfer functions and time domain noise figure described in Section II is used for the simulation. The channel transfer function is alternated between 'narrow region' and 'wide region' within the $\mathrm{AC}$ line cycle. The transmitter and the receiver model in this simulation based on the system model described in Section IV. The following conditions have been assumed in the simulation:

- Ideal synchronization at the receiver;

- The calculated Tone Map(s) is/are totally known both at the transmitter and the receiver;

Fig. 7 shows the effect due to the channel adaptation. As the value of the SNR increases, BER of channel adaptation scenario decreases faster than the BER of the 'no adaptation' scenario. As expected, 'full adaptation' scenario shows the best performance, because the system can fast adapt a sudden change of the channel and noise. In partial adaptation scenario, where $K$ is the number of windows in an AC line cycle, as $K$ increases, the BER performance also increases at the expense 
of the system complexity. It is notable that the performance suffers when there is no AC line cycle based adaptation.

Fig. 8 describes the throughput when the BER is fixed to $10^{-5}$. Obviously, the throughput increases as the value of SNR increases. From the graph, we can observe that AC line cycle based adaptation provide a significant improvement in the throughput. Note that $K$ is about 300 in full adaptation scenario. Much lower value of $K$ compared with full adaptation scenario can improve the system performance. So, if we set $K$ properly, the system throughput can be increased with only a little increase of the system complexity.

\section{CONCLUSIONS}

In this paper, we investigated the performance enhancement that can be achieved by using a channel adaptation mechanism that is synchronized to the AC line cycle. From the measurement, we can confirm that the power line channel alternates two frequency responses within $\mathrm{AC}$ line cycle. Measured powerline noise characteristics are also cyclo-stationary and synchronized with AC powerline. To learn and adapt the cyclic variation of the powerline channel and noise, we propose the channel adaptation process. Analysis of the simulation result for the system based on the Korean powerline standard has shown that a significant improvement in the throughput can be achieved by using AC line based adaptation. Further research incorporating a larger number of channels, as well as taking into account both the frequency response and noise power variation with the AC line cycle, would help to fully understand the potential performance gains offered by the use of line cycle based adaptation in PLC systems.

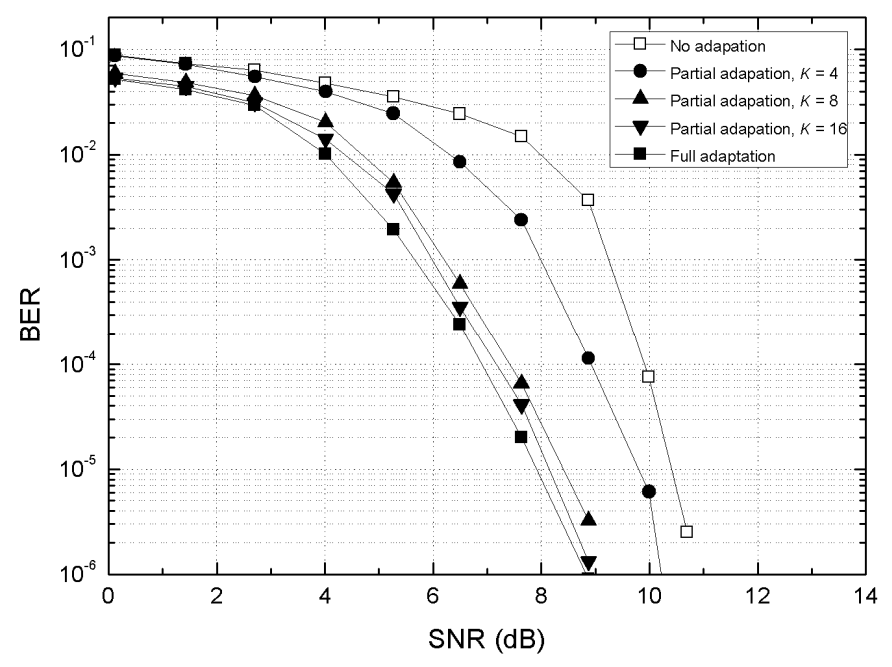

Fig. 7. BER of different channel adaptation scenarios

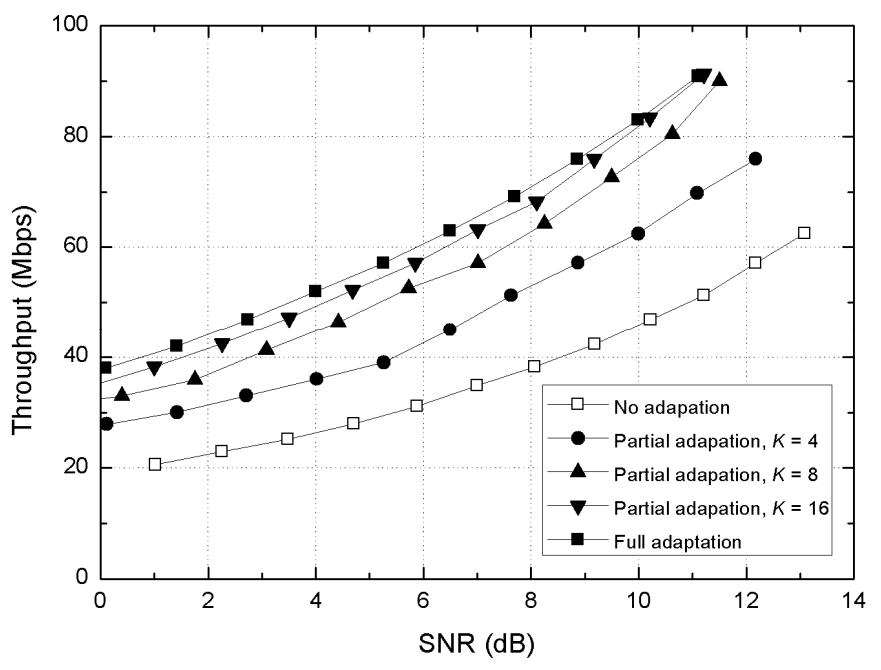

Fig. 8. Throughput of different channel adaptation scenarios

\section{REFERENCES}

[1] M. Zimmermann and K. Dostert, "Analysis and Modeling of Impulsive Noise in Broad-band Powerline Communications," IEEE Trans. Electromagn. Compat., vol. 44, no. 1, pp. 249-258, Feb. 2002.

[2] O. Ohno, M. Katayama, T. Yamazato and A. Ogawa, "A Simple model of cyclo-stationary powerline noise for communication systems," Proc. of the IEEE International Symposium on Power Line Communications and its Applications, Tokyo, Japan, pp. 115-122, Mar. 1998.

[3] M. Katayama, S. Itou, T. Yamazato, and A. Ogawa, "Modeling of cyclostationary and frequency dependent powerline channels for communications," Proc. of the IEEE International Symposium on Power Line Communications and its Application, Limerick, Ireland, pp.123-127, Apr. 2000.

[4] Y. Hirayama, H. Okada, T. Yamazato, M. Katayama, "Noise Analysis on Wide Band PLC with High Sampling Rate and Long Observation Time," Proc. of the IEEE International Symposium on Power Line Communications and its Application, Kyoto, Japan, pp. 142-147, Mar. 2003.

[5] F.J. Canete, J.A. Cortes, L. Diez, J.T.Entrambassaguas, and J.L. Carmona, "Fundamentals of the Cyclic Short-Time Variation of Indoor Powerline Channels," Proc. of the IEEE International Symposium on Power Line Communications and its Application, Vancouver, Canada, pp. 157-161, Apr. 2005.

[6] D. Umehara, T. Hayasaki, S. Denno, and M. Morikura, "The Influence of Time-varying Channels Synchronized with Commercial Power Supply on PLC Equipments," Proc. of the IEEE International Symposium on Power Line Communications and its Application, Jeju, Korea, pp. 30-35, Apr. 2008.

[7] Korean Standard Association, KS X 4600-1: 2006, Information technology - Telecommunications and information exchange between systems - Power Line Communications(PLC) - High speed PLC Medium Access Control (MAC) and Physical Layer(PHY) Part 1 : General requirement, May 2006.

[8] F.J.C. Corripio, J.A.C. Arrabal, L.D. del Rio, and J.T.E. Munoz, "Analysis of the Cyclic Short-Term Variation of Indoor Power Line Channels," IEEE Journal on Selected Areas in Communications, Vol. 24, No. 7, pp. 1327-1338, Jul. 2006.

[9] K. H. Kim, and S. C. Kim, "Performance analysis of LDPC coded systems with bit-loading algorithms for powerline channel," Proc. of the IEEE International Symposium on Power Line Communications and its Application, Pisa, Italy, pp. 234-239, Mar. 2007. 